\title{
Des interfaces pour l'analyse de la signification et du sens
}

\section{Interfaces para el análisis del significado y del sentido}

Jaime Ruiz Vega ${ }^{1}$

\author{
Résumé
}

Dans cet article nous cherchons à aborder des aspects liés aux orientations théoriques qui étudient la signification lexicale et de sens discursif. Nous rendons compte des apports les plus saillants de la Sémantique des Possibles Argumentatifs qui se présente comme un modèle pour analyser la signification des entités lexicales et leur transformation possible à partir de leur insertion dans le discours. Nous traitons également de l'axiologisation discursive qui se traduit en l'évaluation des représentations du monde que construit l'homme et dépasse l'idée de la langue comme permettant seulement la description de ce monde.

\section{Mots-clé:}

Signification, sens, discours, analyse (linguistique) du discours, sémantique des possibles argumentatifs, modalité, modalisation.

\section{Resumen}

En este artículo se tratan aspectos de las propuestas teóricas que estudian el significado léxico y el sentido discursivo. En este texto se da cuenta de los aportes más salientes de la Semántica de los Posibles Argumentativos que se presenta como un modelo para el análisis del significado de las palabras y de su transformación posible a partir de su aparición en el discurso. Se aborda igualmente la axiologización discursiva que se traduce en la evaluación de las representaciones del mundo que construye el hombre y supera, este modelo, la idea de la lengua como un medio que permite simplemente la descripción de ese mundo.

\section{Palabras clave:}

Significado, sentido, discurso, análisis (lingüístico) del discurso, semántica de los posibles argumentativos, modalidad, modalización.

Artículo recibido el 31 de agosto de 2012 y aprobado el 8 de febrero de 2013.

1 Universidad Pedagógica Nacional, Bogotá, Colombia. Correo electrónico: jruiz@pedagogica.edu.co 
La différence entre la signification et le sens a fait lobjet des réflexions et des recherches de plusieurs spécialistes depuis longtemps. Des linguistes et des philosophes se sont intéressés à la problématique de la signification et du sens. Vers 1897 Michel Bréal a jeté les bases de la sémantique en s'intéressant à létude de «la fonction et du sens des mots » (Bréal, 1995, p.1) et a "élaboré une théorie diachronique, axée sur la signification et visant à identifier les causes et les mécanismes des changements linguistiques» (Bréal, p. 1). Depuis lors, affirme Tamba-Mecz, la sémantique se divise en trois périodes : la période évolutionniste, marquée par la sémantique historique; la période mixte, où "s'impose une sémantique lexique mixte, historique et structurale; la période des théories formalisées, caractérisée par les recherches dans le domaine de la sémantique de la phrase et de lénonciation. " (1988, p. 16).

Durant la période des théories formalisées, recouvrant grosso modo les 50 dernières années, se situent, d'après Rastier (2006, p. 5) quatre types d'approches sémantiques. La première, correspondant à la sémantique vériconditionnelle ou sémantique formelle s’est occupée du jugement « de la vérité des énoncés et des conditions auxquelles le langage peut dire le vrai » (Rastier, p. 5). Cette approche considère la signification comme une « relation entre un symbole et l'objet qu'il dénote. » (Rastier, p.5). Comme elle considérait que l' «essence n'est pas une collection de propriétés » (Rastier, p.5), elle n’a vraiment pas développé de sémantique lexicale.

La deuxième approche, la sémantique psychologique, « définit la signification comme le rapport entre des signes et des représentations ou opérations mentales. » (Rastier, p 5). La théorie des conditions nécessaires et suffisantes a favorisé l'apparition de la théorie de la typicalité. Le prototype y est donc apparu comme le représentant d'un ensemble autour duquel sorganisait toute la catégorie. Ce prototype n'est pas le meilleur membre de la catégorie, mais le plus fréquemment utilisé (Kleiber, 1995, p. 48). Le prototype est "l'objet mental, schéma, image cognitive, etc. [...] associé à un mot " (Kleiber, p. 59) et dans cette relation sétablit un processus de catégorisation.
Rastier (2006, p.5) considère la troisième approche, la sémantique cognitive, comme une sorte de développement de la sémantique psychologique $\mathrm{du}$ fait que cette nouvelle approche considère la signification comme une représentation mentale. Ce versant de la sémantique cognitive associe des significations linguistiques à des représentations mentales (Nyckees, 2007). L'une des difficultés de la sémantique cognitive, affirme Rastier (2005), réside dans le fait de considérer les significations comme des représentations et, donc, de les relier à la pensée, un «terme obscur mais commode».

La dernière ou sémantique linguistique autonome s'est fondée sur la linguistique structurale européenne. La signification est le " rapport linguistique entre des signes, et notamment entre des signifiés " car même si les signifiés ont des corrélats psychiques et neuronaux, ces corrélats ne définissent pas ces signifiés (Rastier, 2005). Depuis lors, plusieurs voies se sont développées. Parmi ces branches la Sémantique des Possibles Argumentatifs (désormais SPA) dont nous allons développer plusieurs aspects en rapport avec la problématique de la signification et du sens. Etant l'une des options des sciences du langage qui cherche à aborder autrement la question de la signification et celle du sens, la SPA émerge dans un carrefour d'interfaces permettant d'enrichir l'analyse de la signification des entités lexicales et la manière dont cette signification sactualise dans le discours. Nous allons donc d'abord présenter plusieurs considérations nécessaires à la compréhension de la SPA et de ses postulats.

\section{L'Analyse linguistique du discours}

Galatanu (2003a, p. 214) appréhende l'Analyse du Discours comme l'une des composantes qui, par le biais d'un entrecroisement, permet la proposition et le développement de l'Analyse Linguistique du Discours (désormais ALD). En effet, elle situe l'ALD à la croisée de la Sémantique Théorique et de l'Analyse du Discours. De manière préalable, Galatanu signale que l'Analyse du Discours se trouve face à deux objectifs à atteindre. L'un de ces objectifs est d'« identifier la spécificité du discours étudié » 
id est des invariants d'une pratique discursive, des marques propres d'une identité énonciative ou d'une occurrence énonciative " envisagée dans la singularité de l'acte de parole. » (Galatanu, p. 214)

Le deuxième objectif, à atteindre sur la base des résultats des travaux précédents, consiste à " formuler des hypothèses interprétatives portant sur la pratique humaine qui porte le discours étudié $[. .]$.$» ; ce discours contribue à l'analyse des$ pratiques sociales. Les travaux à effectuer pour atteindre les objectifs définis doivent recourir à des outils linguistiques solides et avoir une approche théorique pouvant rendre compte de la " productioninterprétation du sens discursif en co-texte et en contexte. »(Galatanu, p. 214). Or, cette approche exige une articulation avec une " approche théorique en sémantique $[\ldots]$ susceptible de rendre compte du potentiel discursif des entités linguistiques mobilisées». A partir des précisions sur les deux objectifs précédents, Galatanu en a défini un troisième qu'elle considère fondamental dans ses travaux au sein du GRASP$^{2}$ (Galatanu, 2003a). Ces travaux ont montré la nécessité d'un modèle théorique en sémantique permettant d'expliquer « [...] des effets de sens qui déconstruisent et même intervertissent les valeurs axiologiques $[\ldots]$ des mots » que les dictionnaires présentent comme monovalents. (Galatanu, pp. 213214). Cet objectif contribue au développement de la théorie sémantique et est constitué de deux volets : d'un côté, la proposition de nouvelles hypothèses internes portant sur la signification des entités lexicales et, d'un autre côté, «[...] la validation sur corpus de la description sémantique proposée à partir de ces hypothèses "(Galatanu, 2003a). Lobjectif précédent correspond à ce que Galatanu a appelé Analyse Linguistique du Discours (ALD) et qu'elle a conçu comme « le repérage et l'étude des mécanismes sémantico-discursifs et pragmaticodiscursifs de production et d'interprétation du sens » (Galatanu, 2003a).

GALATANU précise la perspective linguistique de l'analyse du discours quelle propose :

2 Le GRASP, Groupe d'Analyse Sémantique et Pragmatique du Centre d'Analyse Linguistique de Nantes.
Nous allons d’abord définir et décrire l'analyse du discours dans notre perspective linguistique. Cette perspective ne nie pas l'intérêt d'articuler les données sociologiques et psycho-sociales, issues de l'analyse des pratiques sociales et les données linguistiques, issues de l'analyse du produit de l'activité langagière. Elle ne nie pas non plus la démarche la plus courante en analyse $d u$ discours, en tout cas dans l'espace français, qui est de partir de la définition d'un espace social pour étudier les discours qui y sont produits et de revenir sur cet espace pour mieux définir, grâce aux résultats de cette étude, la pratique humaine et les acteurs qui s'y inscrivent. En revanche elle déplace le centre d'intérêt sur létude des mécanismes langagiers qui habilitent le discours à être un terrain privilégié d'influence d'autrui, de présentation de soi, de constructions identitaires, de présentation ou de reconstruction d'un système de valeurs » (Galatanu, 1999a).

D'une certaine manière, on peut considérer que cette orientation de l'analyse du discours proposée par Galatanu se caractérise par ce que Maingueneau appelle une "démarche "intégrative " qui vise à articuler les composants de l'activité discursive, saisie dans sa double dimension sociale et textuelle. » (Maingueneau, 2005).

A partir des considérations précédentes, il est fort important de présenter certaines définitions conventionnelles indispensables à une vue d'ensemble des propositions de Galatanu. Dans cet ordre d'idées, le discours est présenté comme un ensemble d'actes discursifs, id est d'actes de langage qui s'inscrivent dans un champ de pratique, laquelle définit un champ discursif. Autrefois, on confondait les notions de discours et de texte, mais il est clair que dans l'orientation développée par Galatanu, le texte correspond à un ensemble dénoncés qui résultent des actes de langage. Quant aux actes discursifs ou actes de langage, c'est la « réalisation d'une action de nature linguistique (toujours singulière) en contexte (toujours inédit).»

Le schéma ci-dessous rend compte des rapports qui se tissent entre le champ des pratiques humaines et sociales et le champ discursif. La flèche double met en évidence les relations interactives de ces deux champs et de leurs composantes : 


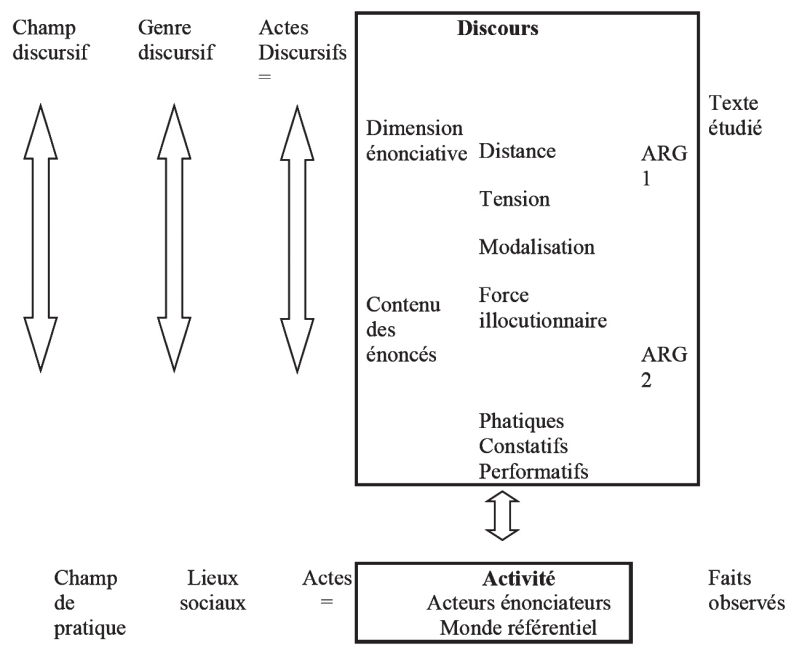

Légende :

ARG 1 = argumentation métadiscursive ARG 2 = argumentation discursive

Figure: Le schéma descriptif (GALATANU, 1996d, 1999a)

Ce schéma descriptif permet d'étudier la complexité des relations qui sétablissent entre les pratiques sociales, y compris les identités et les représentations, et le discours des acteurs sociaux. Ainsi, Galatanu précise que le discours est appréhendé comme :

- voie d'accès privilégiée aux représentations, connaissances et valeurs des sujets, aux identités des acteurs sociaux

- acteur du monde qui agit sur les représentations individuelles pour les conforter, les enrichir, les insérer dans de nouveaux systèmes de connaissances et de valeurs ou encore les déstabiliser, les déconstruire et les reconstruire

- force agissante sur le patrimoine sémantique de la communauté linguistique, mettant en œuvre des mécanismes qui fondent le cinétisme des significations lexicales.

En outre, le schéma condense les composantes de lactivité ou de transformation de la langue en discours d'une manière inédite et particulière, concrétisant de manière permanente des pratiques, lesquelles influencent aussi le discours. De par les entités lexicales utilisées, lesquelles véhiculent des valeurs, et le contexte linguistique, le discours permet au sujet de modifier l'image du monde référentiel, voire des acteurs intervenant dans, ou étant l'objet, des interactions. Devant cette transformation de l'image de soi, de l'autre et du monde opérée au moyen des mots mobilisés dans le discours et qui portent des valeurs, l'ALD s'impose pour tâche première l'identification, la description, la caractérisation et l'explication des mécanismes sémantico-discursifs et pragmatico-discursifs «a) de production de sens, b) d'action sur le patrimoine sémantique de la langue (le cinétisme des significations des entités linguistiques)»(Galatanu, 2007).

D'autre part, dans la même orientation de Benveniste (1966), la dimension énonciative est un paramètre indispensable à l'analyse des marques présentes dans les énoncés. Dans cet ordre d'idées, la distance « représente dans le texte la trace de la prise en charge par le Sujet Communiquant de son statut d'Enonciateur. La distance est minimale si et seulement si, le « je » ou le «nous » ou les autres marques de l'Enonciateur coïncident avec la référence au Sujet Communiquant [...] lêtre social qui produit le discours » (Galatanu, 1997a). Le chercheur peut, à partir de ce concept, déterminer la proximité ou l'éloignement de l'Enonciateur par rapport à son énoncé. Face à cette relation entre l'Enonciateur et son discours, évaluée par la distance, la tension rend compte du rapport entre cet Enonciateur et le destinataire de son discours. Les traces repérées dans le discours permettent dévaluer la construction dont fait l'objet l'interlocuteur et le type d'action que le sujet parlant cherche à exercer sur lui.

Pour ce qui est de la modalisation, Galatanu a recours au concept de modalisation discursive ou "l'inscription dans l'énoncé, par une marque (au sens habituel du mot : trace) linguistique (formes modales), de l'attitude (valeur modale) du sujet communiquant à l'égard du contenu propositionnel de son énoncé et de la fonction que cet énoncé est censé avoir dans l'interaction verbale dont il participe. » (Galatanu, 1997, 2000, 2003). La modalisation discursive peut donc mettre en évidence les valeurs véhiculées dans le discours. De cette manière, le domaine de la modalisation est étudié en termes 
de fonctions discursives, id est représentations, que la «mobilisation de certaines formes linguistiques (formes modales) et la convocation de certaines prises de positions (valeurs modales) rendent possibles. » (Galatanu, 2003).

\section{Les modalités}

Lorsque le chercheur se propose de rendre compte des traces de l'attitude de l'Énonciateur vis-à-vis de son énoncé, il doit avoir recours à la modalité, ce concept qui provient de la logique modale et de la linguistique modale. Ce concept, appartenant à la théorie linguistique, correspond à celui de modalisation dans l'analyse du discours. Puisque la modalisation a déjà été abordée, quelques précisions portant sur la modalité s'avèrent indispensable avant de les aborder dans le cadre de la SPA.

Le concept de modalité a toujours fait partie des préoccupations linguistiques en Occident. Chez les Grecs, notamment dans les travaux d'A ristote, il existait un certain intérêt afin de diviser la proposition en deux parties: " ce dont on dit quelque chose [...] le sujet, et ce qui en est dit [...] le prédicat. » (Chu, 2008, p. 16). À côté de cette différence, Aristote a introduit la dichotomie modus et dictum. Certains énoncés l'ont mené à introduire la proposition modale qui est constituée du modus et du dictum. Lélément modus comprend les modalités : possible, non possible, contingent, non contingent, impossible, nécessaire. Le dictum correspond à l'attribution d'un prédicat à un sujet (Chu, p. 16).

Lauteur fait deux remarques : d'une part, le modus est facultatif dans la proposition et, d'autre part, les modalités concernent le signifié et non le signifiant, cette dernière caractéristique rendant difficile l'identification de l'élément portant le modus dans les énoncés. Cette dernière partie de la proposition peut être représentée par divers éléments et rend encore plus difficile son identification. En outre, le modus et le dictum ont représenté très peu d'intérêt pour les linguistes et c'est Bally qui les a d'abord repris avant d'être imité par Pottier. Il est bon de rappeler que Bally affirme que les phrases peuvent être analysées suivant leur dictum ou " contenu représenté » (propositionnel) et leur modus ou modalité rendant compte de la « position du locuteur par rapport à la réalité du contenu exprimé » (Riegel, Pellat, Rioul, 2004). Pottier fait remarquer qu'à la modalité correspondent plusieurs structures syntaxiques, ce qui rend complexe l'assignation ou l'association de modalités et classe syntaxique. (Chu, 2008).

Mais, au-delà de la préoccupation de Chu provoquée par l'insuffisance de travaux reliant les modalités et la syntaxe, depuis une quarantaine d'années, l'intérêt s'est accru chez divers spécialistes la logique modale étant à la base des études. De même, la sémantique, lénonciation, la pragmatique ont approfondi leurs réflexions dans le domaine des modalités. Surtout, durant ces dernières années, la modalité a été conçue comme une " trace laissée par le locuteur dans l'énoncé » (Chu, p.18). Ces marques du locuteur peuvent apparaître clairement dans lénoncé et la modalité est donc explicite, ou ne pas lêtre, et ce sera une modalité incorporée. En citant Meunier (1974), Riegel, Pellat et Rioul (2004) affirment que l'on a affaire à un " jugement de réalité » lorsque la modalité est complètement absente.

Les réflexions et les travaux menés ces dernières années ont conduit les spécialistes à classer les modalités en modalités d'énoncé et modalités deenonciation. Les modalités dénonciation concernent le sujet de l'énonciation et marquent son attitude énonciative à l'égard de son allocutaire dans ses rapports avec celui-ci, id est " des éléments marquant la force interactive (illocutionnaire) des énoncés» (Galatanu, 2003a). Ce type de modalités est représenté par différents types de phrases, tels les déclaratifs, les injonctifs, les interrogatifs, mais aussi par des adverbes qui qualifient lénonciation qui les contient (García Negroni, Tordesillas Colado, 2001). Dans le cadre de l'analyse des valeurs dans le discours et dans la langue, développée par Galatanu (2003a), ces modalités d'énonciation correspondent à la fonction modale de modalisation dénonciation.

Quant aux modalités d'énoncé, ces traces marquent l'attitude de l'Enonciateur envers le contenu de lénoncé. Riegel, Pellat et Rioul (2004) signalent qu'elles mettent en ouvre la fonction expressive de Jakobson et traduisent l'appréciation que l'Enonciateur porte sur son énoncé. Ces modalités 
rendent compte de la manière dont le sujet parlant se situe par rapport à la vraisemblance, à la vérité, à la certitude, entre autres. Cette attitude du sujet parlant à légard du contenu de son énoncé correspond à la fonction modale de modalisation dénoncé proposée par Galatanu (2003a).

En raison de l'élargissement connu par le concept de modalité dans les années soixante-dix, le terrain de la linguistique modale non seulement a vu étendre son domaine aux aspects précédents, mais il a aussi récupéré des éléments permettant de porter des jugements de valeur sur les prédicats des énoncés produits (qualifiants de noms et caractérisants de verbes), c'est-à-dire sur les modalités de re. A ces modificateurs faisant partie des modalités de re, Galatanu a ajouté des étiquettes nominales et des étiquettes verbales qui portent une valeur modale dans leur signification et n'exigent pas de modificateur pour modaliser l'énoncé qui les véhicule. (cf. Galatanu, 1996, 1999, 2000, 2003). Il en va de même pour ce que Galatanu appelle modalisations discursives ou "l'adhésion ou la non adhésion du sujet parlant par rapport à la source énonciative et la hiérarchisation de l'information dans la phrase » (2003a). Il s'agit donc dans ce dernier aspect d'un autre élément que lélargissement de la linguistique modale a contribué à récupérer.

De ce qui précède, notamment les éléments provenant des travaux et des réflexions de Galatanu, la modalité jouit d'une réorientation favorisée par la sémantique et la pragmatique, notamment par la Sémantique des Possibles Argumentatifs qui voit dans les entités lexicales (formes modales) des unités linguistiques porteuses de valeurs (valeurs modales). Dans cet ordre d'idées, dès que le sujet parlant transforme la langue en discours, il inscrit dans ses énoncés diverses marques qui rendent compte, entre autres, de la transformation de l'image de soi et du monde. A ce sujet, Galatanu conclut ce qui suit :

Par rapport à la définition que nous avons donnée plus haut de la modalisation, entendue comme processus d'inscription d'une attitude, intellectuelle, volitive, évaluative, etc. du "sujet parlant" à l'égard du contenu de son énoncé et du rôle de cet énoncé dans l'interaction verbale, nous envisageons la modalité comme la forme linguistique mobilisée pour exprimer, marquer cette attitude ou valeur modale, comme le résultat linguistiquement marqué du processus de modalisation. C'est au croisement de l'analyse des valeurs modales convoquées et des formes modales (modalités) mobilisées par ces valeurs modales, que l'analyste du discours peut définir et étudier des fonctions modales discursives, c'est-à-dire des processus d'évaluation du monde construit par le discours. (2003a)

\section{La sémantique des possibles argumentatifs}

La Sémantique des Possibles Argumentatifs, proposition que Galatanu a d'abord appelée Sémantique Argumentative Intégrée (Galatanu, 1999, 2000), offre un modèle théorique qui rend compte " des effets de sens qui déconstruisent et même intervertissent les valeurs axiologiques (positives ou négatives) des mots [...]»(Galatanu, 2003b). Ce modèle se propose de réaliser une description de la signification lexicale en articulant l'analyse du discours et la sémantique et il se fonde sur l'idée que la signification des entités linguistiques est une signification que partagent les (inter)locuteurs d'une communauté linguistique, ce qui met en relief le caractère social de la langue. Outre cette dimension sociale, qui permet aux individus d'interagir et de (re)construire la réalité culturelle, la langue se caractérise aussi par ce qu'elle permet au sujet pensant d'exposer sa conception, sa vision du monde, l'idée qu'il se fait des autres, des actions, des interactions. Cette présentation des croyances de chaque individu passe, sur le plan linguistique, tout d'abord par les mots, par ces entités linguistiques dont il dispose et qu'il émet ou insère dans son discours.

Cependant, le locuteur qui parle du monde, qui en rend compte dans son interaction ne se limite pas à le décrire, à narrer ce qui s'y passe, à exposer des caractéristiques des objets et des interactions de la réalité. Il ne se contente pas de porter un regard et de dire d'une manière neutre, mais il évalue ce monde et les relations qui s'y établissent, les autres et leurs interactions. Le sujet de par son insertion 
dans la communication véhicule des jugements qui sont portés par les entités lexicales qu'il utilise dans son discours. Dans ce sens, la SPA veut être « une théorie sémantique susceptible de rendre compte du niveau d'inscription de la valeur axiologique dans la signification des mots. "(Galatanu, 2003). En guise d'exemple, lorsqu'un locuteur affirme : " À la différence d'Occident, dans d'autres cultures 'non occidentales' on respecte la femme ", il faut déterminer ce qu'il entend par le respect, les stéréotypes linguistiques associés à ce mot et les valeurs portées. En outre, il faut prendre en considération s'il s'agit d'un Français, d'un Colombien ou d'un locuteur appartenant à l'une de ces cultures « non occidentales ».

\section{Un carrefour d'interfaces}

La SPA se veut une approche de la signification lexicale et du sens discursif et se situe dans le champ disciplinaire formé par un carrefour de quatre interfaces (Galatanu, 2009a, p. 50) :

Sémantique lexicale - Sémantique textuelle Sémantique textuelle - Analyse du discours Sémantique lexicale - Analyse du discours Sémantique lexicale - Analyse linguistique du discours

Dans ce carrefour, la SPA construit la rencontre des quatre interfaces citées ci-dessus et qui sont caractérisées par les aspects qui suivent :

L'Analyse du discours qui est envisagée par la SPA comme « l'étude d'une pratique de construction langagière de soi et du monde, productrice de son propre univers » (Galatanu, p. 50).

L'Analyse linguistique du discours ou " l'étude des mécanismes discursifs (sémantiques et pragmatiques) de construction du sens »(Galatanu, p. 50).

La Sémantique lexicale "appréhendée comme un modèle de la description de la signification lexicale, permettant de rendre compte à la fois de son ancrage dénotatif et de son potentiel argumentatif, notamment axiologique » (Galatanu, p. 50).

La Sémantique textuelle, envisagée en tant qu'un "modèle de description des traces, dans le texte, des mécanismes discursifs de construction du sens " (Galatanu, p. 50).
Inspirée par les idées de Putnam (1975, 1990, 1994 (Galatanu)) sur la signification des mots, et dans la filiation de la sémantique argumentative (Anscombre, Ducrot, Carel), l'approche proposée par la SPA se veut holistique, associative et encyclopédique. A partir des propositions de Putnam qui présentent la signification en termes de noyau et de stéréotypes, Galatanu a développé un modèle de description de la signification lexicale à trois strates: noyau, stéréotypes et possibles argumentatifs, qu'elle a définis et décrits dans une perspective " argumentativiste » : le noyau, les stéréotypes et les possibles argumentatifs. A ces strates de la signification lexicale, elle a ajouté une forme de manifestation discursive, les déploiements argumentatifs, qui situent le modèle à l'interface de la langue et du discours. Prenons, par exemple, le mot français échec : à partir de l'analyse du discours définitionnel lexicographique dans deux dictionnaires, nous obtenons que son noyau (N) correspond à : Vouloir un résultat $\mathrm{X}$ bon $\mathrm{DC}$ chercher un résultat $\mathrm{X} \mathrm{DC}$ effort PT obtenir un résultat $\mathrm{X}$ mauvais. Parmi les stéréotypes (St), nous avons identifié ce qui suit : N DC volonté/but/initiative/tentative/DC ne pas aboutir... PT affermissement des forts. La troisième strate (PA) de cette description se présente comme suit : échec DC volonté/but/initiative/tentative/DC ne pas aboutir... PT affermissement.

En tant qu'approche associative et holistique de la signification, la théorie spéaienne prend les stéréotypes d'une entité lexicale comme des représentations des " associations, dans des blocs de signification argumentative (relation posée comme une " relation naturelle " : cause-effet, symptômephénomène, but-moyen, etc.) des éléments du noyau avec d'autres représentations sémantiques. » (Galatanu, 2005a, p. 57). Lidentification concrète des bornes rigides aux ensembles ouverts, formés par ces associations « relativement stables » à l'intérieur d'une communauté linguistique à un moment donné de lévolution de sa langue s’avère impossible. A titre d'exemple, les éléments du $\mathbf{N}$ du mot succès DC obtenir X DC approbation/célébrité sassocient aux stéréotypes DC bonheur/prospérité au moyen de rapports de cause-effet. 
A partir de cette condition, l'approche de la SPA se définit comme encyclopédique « car tous les aspects de notre connaissance de l'entité en jeu contribuent au sens de l'expression qui la désigne. » (Galatanu, p. 57). Et, de façon plus générale, toutes les connaissances que nous aurons intégrées et qui font partie du capital individuel et culturel nous permettant de naviguer dans la toile socioculturelle et interculturelle par le biais, entre autres, des langues, contribuent à l'analyse de la signification lexicale. A ce sujet, Galatanu (2005d, p. 193) établit que « Tous les aspects de notre connaissance participent également à la reconstruction discursive de la signification lexicale grâce au phénomène de 'décohérence' et de 'contamination' sémantique et/ ou pragmatique ».

Rappelons que la Sémantique des Possibles Argumentatifs se présente comme un modèle qui se fonde sur trois hypothèses et qui est constitué de trois strates et « une forme de manifestation discursive, donc 4 niveaux » (Galatanu, 2009b). A ce que nous venons de dire, il faut ajouter que dans la même orientation de la sémantique argumentative prônée par Anscombre et Ducrot, entre 1983 et 1995, le modèle SPA pose comme postulat que le sens des énoncés est argumentatif et établit comme hypothèse que ce sens " s'appuie sur et (ré)inscrit avec chaque occurrence discursive, un potentiel axiologique (donc argumentatif) dans la signification des entités lexicales » (Galatanu, 2009b). Cette hypothèse renvoie au fait que l'homme inscrit sa perception du monde dans les entités lexicales et, orienté d'une certaine manière par sa culture, par ses expériences, son vécu et sa formation, évalue ce monde et les relations qui y ont lieu. De cette façon, il renforce ou actualise la signification de ces entités linguistiques dans l'action discursive. Mais, même avant la mise en discours de ces entités lexicales, l'individu peut faire changer la signification qu'il accorde aux mots dont il se sert pour s'insérer dans la réalité.

La deuxième hypothèse établit que le potentiel axiologique des entités linguistiques existant sous forme "d'associations dans des blocs sémantiques d'argumentation »(Galatanu, 2009b) se caractérise par son ancrage culturel. Cette base culturelle lui accorde aussi son caractère évolutif et à la signification lexicale un cinétisme même si le noyau est épargné de modifications en raison de ses caractéristiques fondées sur des universaux et des primitifs sémantiques ou tel qu'il est présenté par Galatanu (2009a) : « [...] un noyau stable, que nous pouvons représenter en termes d'universaux et de primitifs sémantiques $[\ldots]$ ».

L’ancrage dénotatif, référentiel, apparaît dans le noyau et dans les stéréotypes, ces derniers étant ancrés culturellement et, donc, évolutifs. Cette troisième hypothèse a pour cadre le langage « appréhendé comme outil cognitif, de représentation du monde "perçu et modélisé " (Kleiber, 1999) par la langue, au travers " des significations linguistiques »(Galatanu, 2009b). C'est l'association des représentations du noyau, et de ses éléments, avec celles des stéréotypes qui produit la dimension argumentative. Elle se manifeste aussi dans des blocs d'argumentation externe id est au niveau de possibles argumentatifs ou de séquences discursives formées par l'association de l'entité lexicale avec des éléments de son stéréotype.

Dans les lignes précédentes nous avons exposé les hypothèses qui présentent le potentiel argumentatif des entités lexicales, fondé sur une base culturelle et marqué par son caractère évolutif et structuré par les associations du noyau avec des stéréotypes dans des blocs d'argumentation interne ou par des relations avec des représentations d'autres entités linguistiques dans des blocs d'argumentation externe. À partir de ce cadre, la SPA présente le modèle qui rend compte de la description de la signification lexicale sous forme d'un modèle, rappelons-le, formé de 4 niveaux : 3 strates et une forme de manifestation discursive, à savoir : le noyau, les stéréotypes, les possibles argumentatifs et les déploiements argumentatifs.

Le noyau $(\mathrm{N})$ correspond aux traits de catégorisation sémantique décrits par Putnam en 1975 comme lélément stable de la signification lexicale. La SPA a considéré ces traits comme « des propriétés essentielles »(Galatanu, 2009b, p. 394). En guise d'exemple, nous présentons ci-dessous la description 
de la signification lexicale de science, notamment de son noyau, effectuée par Nikolenko $(2011,91)$ :

«Science, propriétés essentielles :

Type grammatical : nom, féminin

Zone modale: épistémique

Orientation axiologique : positif

Ensemble de connaissances et d'expériences objectives et vérifiables / organisées / structurées / qui ont un objet /une méthode

Connaissances expérimentales ou livresques DC savoir exact/ loi/ fait [...]»

Les stéréotypes (St) sont envisagés par la SPA comme « un ensemble ouvert des éléments du noyau avec d'autres représentations, constituant des blocs d'argumentation interne » (Galatanu, p. 395). Un cas de figure est la description de la signification du mot harmonisation (Ignatieva Chaillou, 2011, p. 149) dont les stéréotypes apparaissent ci-dessous :

N DC

«orienter les lois vers un objectif commun / former un tout cohérent /concourir à une même fin / établir un ordre / mettre en équilibre [...] / finalité commune / cohérence / équilibre / coordination...»

Il est à remarquer que le caractère ouvert de l'ensemble est représenté par les pointillés (...).

Les possibles argumentatifs (PA) tirent leur existence de l'association de l'entité lexicale avec l'un des éléments de son stéréotype et correspondent à des séquences discursives qui sont calculées à partir des stéréotypes constituant cette unité. Ces probabilités argumentatives prennent place dans des blocs d'argumentation externe. Ces associations virtuelles « sorganisent dans deux faisceaux orientés respectivement vers l'un ou l'autre des pôles axiologiques (positif et négatif) »(Galatanu, 2005a). L’environnement sémantique ou le contexte produisent une contamination discursive qui entraîne, à son tour, lorientation positive ou négative du faisceau d'associations. Suivant leur organisation dans deux

faisceaux, les possibles argumentatifs peuvent être conformes ou non-conformes à l'orientation axiologique du stéréotype. Nous faisons remarquer, dans l'exemple suivant (Galatanu, 2009b), que les stéréotypes ont des rapports avec les éléments du noyau du mot, alors que les PA correspondent à des associations de ces stéréotypes avec l'entité lexicale elle-même :

\section{Travail}

N

Nécessité
Et/ou
Obligation de fair
utilement et
et faire utilement
DC
Effort
DC
Résultat

Les déploiements argumentatifs (DA), ou forme de manifestation discursive, "sont les séquences argumentatives réalisées par les occurrences discursives " (Galatanu, 2009b). Ce niveau, qui n'est présent que dans le discours, vise l'actualisation des possibles argumentatifs. Ces possibles argumentatifs permettent de mettre en rapport le sens des occurrences discursives et la signification de l'unité lexicale et de vérifier, par la suite, la conformité ou la non-conformité de ce sens au protocole sémantique de la signification. Galatanu (2009a, p. 62) rend compte d'un mécanisme qui transgresse le protocole sémantique du mot belle dans lénoncé «Elle est belle, pourtant elle n'est pas superficielle. » Elle affirme qu'il s'agit dans ce cas d'un phénomène d'insertion discursive d'un nouveau stéréotype dans la signification du terme étudié. 


\section{Le dispositif de signification}

Dans cette approche de la SPA, un dispositif de signification est à l'origine des possibles argumentatifs et il est à son tour formé par le noyau et les stéréotypes des entités lexicales analysées. Ce dispositif se construit «à partir des énoncés définitionnels lexicographiques et des exemples proposés par les dictionnaires »(Galatanu, p. 62). Quant aux déploiements argumentatifs, ils existent dans un contexte par le biais des occurrences discursives. C'est donc la mise en discours des entités lexicales et leurs interactions qui font apparaître les déploiements argumentatifs, ce qui présente le discours comme un phénomène observable revendiqué par la SPA.

Or, le modèle de la SPA s'impose quatre conditions qu'il doit observer afin de mener à bien la représentation sémantique :

" cette représentation doit comporter une dimension descriptive de la signification lexicale, préservant ainsi le recours à une lecture de la forme logique de la phrase énoncée;

elle doit faire apparaître à la fois la "partie stable » de la signification et la partie évolutive, que le discours proposé charge et/ou décharge de valeurs ;

rendre compte du statut d'ensemble ouvert des éléments de signification évolutifs ou " stéréotypes »;

rendre compte du potentiel discursif (argumentatif) de la signification lexicale. »

Sur la base des hypothèses abordées ci-dessus, ainsi que des strates et des conditions qu'elle définit, la SPA fournit un «modèle de la construction de la signification lexicale ou bien de la reconstruction discursive de la signification lexicale à partir des hypothèses émises dans et par l'interprétation du sens de différentes occurrences d'emploi des lexèmes concernés »(Galatanu, 2007a, p. 314).

\section{La signification lexicale à la lumière de la sémantique des possibles argumentatifs}

D’une manière générale, l'utilisation que le locuteur fait des entités lexicales et l'insertion de celles-ci dans le discours est l'objet de la SPA. C'est ainsi que la description de la signification lexicale se présente comme une construction théorique fondée sur les emplois de ces entités et l'analyse du sens construit dans le discours. Des hypothèses internes et externes se croisent pour bâtir une interface à partir de laquelle se relient deux voies permettant la construction de la signification.

Les hypothèses internes concernent la construction de la signification lexicale à partir des discours lexicographiques, soit les discours définitionnels que constituent les dictionnaires. Par conséquent, le chercheur se consacre à l'identification et à l'étude des entités linguistiques telles qu'elles apparaissent dans les dictionnaires et, de cette manière, il rend compte des aspects stables de ces unités, les noyaux, et de leurs stéréotypes. A ces deux strates, s'ajoutent les possibles argumentatifs. Les analyses menées à la lumière de ces hypothèses conduisent à la construction linguistique de la signification des mots. Voici un exemple de la première étape effectuée pour la construction de la signification lexicale du terme succès. Pour ce faire nous avons eu recours aux dictionnaires Le Nouveau Petit Robert 2009 (NPR2009) et le Lexis Larousse de la Langue française (LLLF) :

Le NPR2009 définit l’entité lexicale succès comme suit:

1. Vx Ce qui arrive de bon ou de mauvais à la suite d'un acte, d'un fait initial.

2. Mod. Heureux résultat (d'une décision, d'une entreprise, d'une suite d'évènements); caractère favorable de ce qui arrive.

3. Le fait, pour qqn, d’obtenir ce qu'il a cherché, de parvenir à un résultat souhaité

4. Évènement particulier, circonstance qui constitue un résultat très heureux pour qqn.

5. Le fait d'obtenir une audience nombreuse et favorable, dêtre connu du public.

6. Le fait de plaire. [...]

Les définitions du succès proposées par le LLLF correspondent à ce qui suit : 
1. Résultat heureux obtenu dans une entreprise, dans une affaire, dans un travail.

2. Approbation du public. [...] Ruiz $(2011,121)$

Cest à partir de létude de ces définitions dictionnairiques que le chercheur établit le $\mathrm{N}$ et les St. de l'entité lexicale étudiée.

Les hypothèses externes correspondent aux occurrences discursives, id est la mise en discours des entités lexicales et la génération de multiples modifications en raison de la contagion sémantique et de l'insertion des discours dans les différents domaines d'interaction socioculturelle des usagers de la langue. Dans ce cadre, les déploiements argumentatifs permettent de confronter les stéréotypes des entités linguistiques à leurs stéréotypes et de rendre compte donc de la conformité ou de la nonconformité au protocole sémantique. C'est à partir de la collecte de données, par le biais de questionnaires par exemple, que le chercheur a accès à des corpus lui permettant d'y porter des analyses et de reconstruire, sur le plan discursif, la signification des mots. Galatanu (2003a, p. 220) propose l'exemple qui suit pour illustrer la modification dans la signification du mot colère, dans l'un de ses stéréotypes, ce qui intervertit le pôle axiologique au niveau de cette strate : «Il est en colère, mais il est lâche, donc je ne l'estime pas ». Dans cet énoncé le stéréotype axiologiquement négatif " agir violemment ", associé à lélément du $\mathrm{N}$ « éprouver émotion/sentiment négatif violent », est mis en rapport avec le courage, ce qui rend le stéréotype axiologiquement positif.

\section{Aspects de la signification lexicale}

Comme il a déjà été avancé, la SPA propose un modèle de description de la signification qui peut rendre compte du langage comme outil argumentatif à double dimension : l'une cognitive, l'autre expressive ou communicative. La SPA analyse le langage dans sa dimension cognitive à partir de la description des représentations que les locuteurs se font du monde par le biais de la langue. Grâce à la langue, l'individu perçoit et modélise la réalité et en rend compte. De la même manière, le modèle sémantique permet d'étudier le langage comme outil d'expression au moyen de la description de "l'expression de soi et de communication et, par voie de conséquence, d'argumentation de soi et du monde représenté » (Galatanu, 2007a)

En prenant en considération le cognitif et l'expressif/argumentatif du langage actualisé dans la langue et donc, initialement, dans le lexique, le modèle de la SPA soccupe de la signification en tant que dénotation ou description, d'une part, et en tant qu'argumentation, d'autre part. Le processus de sémiotisation qui permet de représenter le monde " perçu et modélisé par la langue » est à l'origine du caractère dénotatif ou descriptif. Pour ce qui est du caractère discursif ou argumentatif, c'est le processus d'argumentation qui le forge lorsqu'il contribue à l'évaluation des représentations du monde, durant la communication.

Dans cet ordre d'idées, puisque la description de la signification lexicale doit être en mesure de rendre compte de cet aspect dénotatif ou descriptif, cette signification se présente comme "une conceptualisation largement identique d'un individu à l'autre, ce qui forme une sorte de socle pour une intercompréhension réussie» (Kleiber, 1999) ayant une fonction de "stabilisation du monde dans la langue" »(Galatanu, 2003, p. 215).

La description de la signification lexicale doit aussi rendre compte du potentiel argumentatif des entités lexicales et le " niveau d'inscription de ce potentiel dans la signification lexicale » (Galatanu, p. 215). Les enchaînements argumentatifs des énoncés actualisent le potentiel et s'investissent d'une dimension évaluative des représentations du monde qu'ont les individus.

La SPA s'impose également pour tâche l'étude $\mathrm{du}$ « fonctionnement discursif polyvalent de ce potentiel argumentatif dans l'interaction avec l'environnement sémantique et/ou le contexte pragmatique » en raison du fait que ce potentiel argumentatif ou modal " peut être activé, voire renforcé ou au contraire, affaibli, voire neutralisé ou même interverti »(Galatanu, 2007). Voici des exemples de ces mécanismes (fondés sur la signification des termes mobilisés et/ou la «contamination séman- 
tique " (Galatanu, 2009a, p. 54) du co-texte) : Elle est intelligente, elle pourra s'en sortir rapidement (activation) ; Il est vraiment bête, il n'a rien compris (renforcement); C'est un crime, mais il y a des circonstances atténuantes (Galatanu, p. 54) (affaiblissement); Soyez raisonnable, achetez-vous une voiture de luxe (Galatanu, 2009a, 63) (interversion).

\section{La Sémantique des Possibles Argumentatifs et la signification}

Comme nous l'avons signalé auparavant, les qualités conventionnelle et durable de l'association qui sétablit entre une représentation et une expression verbale caractérisent la signification à la lumière de la SPA. Avant d'aborder la présentation du modèle de cette dernière, il est indispensable de préciser ce qu'est la signification pour cette théorie sémantique. Galatanu (2000, p. 29) conçoit comme étant la signification :

La /les représentation(s) associée(s) à une expression verbale de façon durable dans une communauté linguistique (par convention de l'institution langagière au sens de Searle) (Searle, 1969)) et correspondant plus ou moins ou tout au moins pour la place qu'elle occuperait dans le triangle d'Ogden et Richards) au signifié saussurien - 1916/1968), à l'interprétant de Peirce (1830-1914/1978), à la référence d'Ogden et Richards (1923), à la désignation de Morris (1938).

\section{La sémantique des possibles argumentatifs et l'axiologisation discursive}

Lorsque le chercheur analyse les propos tenus par un individu, qu'ils soient présentés à l'oral ou par écrit, il ne cherche pas à décider du caractère vrai de ses propos mais de la manière dont il se représente le monde. A ce sujet, Dervin, dans son article sur des approches dialogiques et énonciatives de l'interculturel affirme que «L'analyse du discours ne cherche pas à savoir si tel ou tel énoncé est véridique mais elle s'intéresse avant tout aux représentations qui traversent les discours.» (2009, p. 170).

En reliant les modalités et l'activité de l'enseignant en classe de langue étrangère, Dervin rappelle que Le Querler insiste sur l'importance d'aborder les modalités afin de favoriser le développement d'une identité mouvante :

Le Querler, dans son ouvrage Typologie des modalités (1996), propose de s'intéresser aux modalités aléthiques (possible, impossible, nécessaire et contingent), modalités déontiques (devoir), modalités temporelles, modalités subjectives (épistémiques), modalités intersubjectives et modalités implicatives (une proposition en implique une autre). C'est à travers ces éléments que l'on pourra noter la mise en scène de soi et de l'Autre et donc la co-construction de l'identité mouvante des sujets dans un contexte interculturel. En apprenant à relever ces traces énonciatives, l'apprenant peut être amené à mieux contrôler son propre discours, éviter la mise en scène différentialiste-culturaliste, avoir un impact sur les attitudes, et ainsi agir/réagir éthiquement face à l'altérité. (2009, p. 170)

Bien que ces propositions contribuent à la construction d'une compétence interculturelle et, par effet ricochet, à l'intercompréhension, il s'avère important de proposer des démarches visant à développer de manière fondamentale les compétences sémantique et pragmatique. Dans cette orientation le traitement des modalités permet aux apprenants de façonner une compétence de communication plus solide dans laquelle ils seront capables de différencier clairement les valeurs mobilisées dans leurs discours et dans ceux d'autrui. Autrement dit, il serait capable de faire des échanges aux cours desquels les entités linguistiques qu'il émet ou qu’il entend correspondent exactement aux significations à la lumière de chacune des cultures intervenant dans la communication à un moment donné.

\section{L'axiologisation discursive}

Il est important de rappeler que le langage actualisé dans la langue permet non seulement la description du monde, mais surtout l'évaluation des représentations du monde que construit l'homme. Le locuteur inscrit donc dans son expression, à commencer en premier lieu dans les entités lexicales -de par leur potentiel argumentatif -, «[...] des évaluations, liées, 
référées à des champs d'expérience humaine [...]» (Galatanu, 2003, p. 217). Il existe donc des mécanismes langagiers au moyen desquels l'homme se construit lui-même et construit le monde, d'une part, et porte des évaluations sur soi-même et sur le monde qu'il a construit, d'autre part. Cette construction et cette évaluation se réalisent dans et par le discours. Galatanu établit l'hypothèse que « Le discours, même s'il n'a pas pour objet les valeurs, des problèmes d'éthique, de morale, de déontologie, ou pour objectif la transmission, le renforcement ou encore la reconstruction d'un système de valeurs, n’en est pas moins évaluatif de la représentation qu'il donne du monde et, de ce fait, porteur de valeurs. » (2003b, p. 87)

Dans cet ordre d'idées, l'analyse du discours permet de rendre compte aussi bien de 1) «la présence des systèmes de valeurs " dans les discours descriptifs, analytiques et théoriques sur les valeurs, et dans ceux qui ont pour objet la transmission de ces valeurs, que de 2) la « dimension édifiante » présente dans n'importe quel type de discours. (Galatanu, 2003b, p. 88). Dans le premier cas, il s'agit des discours que Galatanu appelle édifiants et qui ont pour objet les valeurs et pour but leur préservation ou leur acquisition. De même, ce cas concerne les discours qui portent « sur les valeurs, sur leurs origines, leur respect et leur transgression » (Galatanu, p. 88), sans pour autant chercher à les préserver, les transformer ou les (faire) respecter. C'est donc à ces deux niveaux que les valeurs font l'objet d'étude du linguiste et auxquels il faut ajouter un troisième portant sur le caractère argumentatif des discours : "La dimension argumentative de tous les discours, inscrite dans la signification des unités lexicales mobilisées ou construite en contexte, autorisant une orientation axiologique (négative ou positive) des enchaînements discursifs qu'il rend possible. » (Galatanu, p. 88)

Dans son texte "La construction discursive des valeurs ", Galatanu (2003b, p. 89) aborde trois sources de sa réflexion sur le sujet qui loccupe. La première étant la diversité de discours sur les valeurs dans toutes les pratiques sociales ce qui fournit au linguiste des corpus riches et variés qui rendent compte de la déconstruction et reconstruction des systèmes de valeurs et qui permettent d'étudier les liens entre les pratiques humaines et les discours qui le portent. Elle affirme que cette réalité invite le chercheur à reposer la problématique des valeurs (définition, origine, généralité, universalité et circulation des systèmes de valeurs) dans une perspective linguistique.

La deuxième source concerne le discours comme porteur de valeurs du fait qu'il favorise non seulement la construction de l'individu et du monde, mais aussi l'évaluation de ces résultats de la construction. Ainsi, le discours axiologise ce monde construit. Le discours étant porteur de valeurs devient l'objet d'analyse du linguiste. Pour ce faire, le linguiste s'impose comme tâche la formulation d'hypothèses sur la nature édifiante du discours, son caractère évaluateur et "sur les mécanismes qu'il met en œuvre pour 'axiologiser' le monde qu'il construit » (Galatanu, p. 89). En conséquence, dans le cadre de la SPA, s'examinent, comme il a été fait ailleurs, les concepts de modalisation et valeur modale proposés par la linguistique afin détudier la prise de position du sujet énonciateur dans et par son discours. Dans ce sens et dans le domaine de la sémantique, le modèle de la SPA est en mesure de démontrer que les entités lexicales peuvent avoir une ou plusieurs valeurs modales parmi les éléments de leurs stéréotypes ou même dans leurs noyaux. (Galatanu, 1999)

Quant à la troisième source, en rapport avec la dimension argumentative de tous les discours, elle correspond à « l'interrogation sur le fonctionnement des entités lexicales dans la construction du sens discursif, en lien avec un modèle de description en sémantique lexicale » (Galatanu, 2003b, pp. 88-89), et aussi à l'interrogation sur les concepts utilisés dans la description du potentiel argumentatif du langage. Il en découle l'exigence d'une articulation de l'analyse du discours avec une approche sémantique théorique pouvant soccuper du potentiel axiologique des mots. Aussi, est-il important de rappeler que Galatanu se propose de revoir le concept de modalité et de soutenir " une 'entrée' sémantique dans ce domaine de la linguistique. » (Galatanu, p. 89) 
Une recherche scientifique de la signification lexicale et des valeurs mobilisées en langue maternelle ou en langue étrangère, chez n'importe quel type de locuteurs, se situe dans le domaine linguistique et il s'avère obligatoire de recourir à une approche linguistique de la notion de valeur. Cette approche linguistique, développée à la lumière de la sémantique argumentative, s'appuie sur trois concepts:

celui de modalisation qui relève de l'analyse du discours,

celui de modalité faisant partie de la théorie linguistique des modalités,

celui de valeur modale « relevant d'une approche sémantique des modalités (Le Querler, Galatanu, 2002)» (Galatanu, 2003b, p. 89)

Galatanu définit la modalisation discursive comme "L'inscription dans l'énoncé, par une marque ([...] trace) linguistique (formes modales), de l'attitude (valeur modale) [intellectuelle, volitive, évaluative] du sujet communiquant à l'égard du contenu propositionnel de son énoncé et de la fonction que cet énoncé est censé avoir dans l'interaction verbale dont il participe. (Galatanu, 1997, 2000) » (Galatanu, 2003b, p. 90).

C'est ainsi que la mobilisation des formes linguistiques ou formes modales et la convocation de quelques prises de position ou valeurs modales favorisent l'apparition de fonctions discursives qui actualisent la modalisation. Galatanu souligne que cette définition doit prendre en considération celles de valeur provenant des sciences humaines et sociales et qui rendent compte de sa charge subjective. Dans ce sens, la valeur que l'individu accorde à un aspect du monde est fondée sur l'intérêt, la rupture de l'indifférence, un choix, une affinité et un parti pris. Cette valeur est liée à la conception du monde du sujet pensant et agissant et, comme le signale Galatanu « toute valeur s'inscrit ainsi sur l'axe positif/négatif pour évaluer et hiérarchiser les choses » et « les valeurs ont une charge positive " (Galatanu, p. 91) et renvoient à un idéal, au bien et même à la norme, lesquels peuvent changer suivant la culture et en fonction de leur forme et de leur contenu.

Devant cette importance des valeurs, le chercheur doit étudier leur énonciation par le biais de « mécanismes argumentatifs mettant en œuvre des valeurs inscrites de façon relativement stable dans la signification des mots [...]»(Galatanu, p. 91). Mais ces mécanismes peuvent aller plus loin, dans la mesure où ils peuvent déconstruire la signification des entités lexicales porteuses de valeurs axiologiques et en même temps les affaiblir, les neutraliser, les intervertir. Les travaux de Galatanu ont permis de mettre en relief tant le rôle du langage dans l'émergence des valeurs que les «mécanismes de propagation des systèmes de valeurs »(Galatanu, p. 92).

Tel que nous l'avons exposé auparavant, la SPA met en exergue trois concepts fondamentaux pour l'analyse des valeurs dans le discours et dans la langue. Le concept de modalisation, du domaine de l'analyse du discours, correspond à celui de modalité dans le champ de la théorie linguistique. Relevant de la logique et de la linguistique modales, tout comme le concept de valeur modale, celui de modalité s'est développé et son ampleur a permis de passer de la prise en considération de l'attitude du sujet à l'égard du contenu de lénoncé (et qui équivaut en SPA à la modalisation d'énoncé) à la récupération des éléments qui marquent la force illocutionnaire des énoncés (et qui correspondent en SPA à la modalisation d'énonciation) et de nombreux éléments qui traduisent le jugement de valeur que portent les sujets parlants sur les prédicats des énoncés : qualifiants des noms et caractérisants des verbes (modalités de re). Dans ces dernières, Galatanu a inséré aussi les prédicats nominaux (des noms) et verbaux (des verbes ou expressions verbales) qui véhiculent une valeur modale dans leur signification et peuvent modaliser l'énoncé pour la pure et simple raison d’y apparaître. (Galatanu, pp. 92-93)

Pour ce qui est de la modalité, elle correspond à la «forme linguistique mobilisée pour exprimer, marquer cette attitude [du sujet parlant] ou valeur modale, comme le résultat linguistiquement marqué du processus de modalisation »(Galatanu, p. 93). 
Dans cet ordre d'idées, les trois concepts peuvent être représentés ainsi :

Modalisation : processus d'inscription

Modalité : forme linguistique mobilisée, forme modale

Valeur modale : attitude, prise de position

Létude que mène le chercheur afin de rendre compte de l'évaluation du monde résultant de l'action discursive (fonctions modales discursives) prend en considération l'intersection de l'analyse des formes modales que le locuteur mobilise, id est les modalités de re et de dicto, et des valeurs modales portées par ces formes (entités lexicales). Galatanu propose dans le tableau ci-dessous un résumé des fonctions modales discursives qui résultent de croiser les contenus sémantiques mobilisés porteurs de valeurs modales et les formes linguistiques mobilisées :

\section{La modalisation discursive}

\begin{tabular}{|c|c|c|}
\hline $\begin{array}{c}\text { Fonction } \\
\text { modale }\end{array}$ & $\begin{array}{l}\text { Modalisation ud'énoncé» } \\
\text { = inscription dans le discours de } \\
\text { l'attitude du sujet } \\
\text { communiquant à l'égard du } \\
\text { contenu de son énoncé }\end{array}$ & $\begin{array}{l}\text { Modalisation "d'enonciation" } \\
=\text { inscription dans le discours de } \\
\text { l'intention illocutionnaire du sujet } \\
\text { communiquant }\end{array}$ \\
\hline $\begin{array}{l}\text { Valeurs } \\
\text { modales }\end{array}$ & $\begin{array}{l}\text { - valeurs ontologiques } \\
\text { - aléthiques } \\
\text { - déontiques } \\
\text { - valeurs de jugement de vérité } \\
\text { - épistémiques } \\
\text { - doxologiques } \\
\text { - valeurs axiologiques } \\
\text { - éthiques } \\
\text { - morales } \\
\text { - esthétiques } \\
\text { - pragmatiques } \\
\text { - intellectuelles } \\
\text { - hédoniques } \\
\text { - affectives } \\
\text { - valeurs finalisantes } \\
\text { - volitives } \\
\text { - désidératives }\end{array}$ & $\begin{array}{l}\text { Configurations de valeurs modales } \\
\text { spécifiques des classes d'actes } \\
\text { illocutionnaires }\end{array}$ \\
\hline $\begin{array}{l}\text { Formes } \\
\text { modales }\end{array}$ & $\begin{array}{l}\text { - modalités de re } \\
\text { - modalités de dicto }\end{array}$ & $\begin{array}{l}\text { Modalités illocutionnaires = marques } \\
\text { linguistiques des intentions } \\
\text { illocutionnaires }\end{array}$ \\
\hline
\end{tabular}

Tableau : La modalisation discursive (Galatanu, 2003, p. 94)

\section{Les zones sémantiques modales}

Rappelons que dans l'approche linguistique des valeurs proposée par Galatanu l'un des concepts clés est celui de valeur modale qui équivaut à l'attitude du sujet (pensant, parlant et agissant) vis-à-vis du « contenu propositionnel de son énoncé et de la fonction que cet énoncé est censé avoir dans l'interaction verbale dont il participe. (Galatanu, 1997, 2000).» (Galatanu, 1999, p. 90). Ces valeurs modales se matérialisent dans les formes modales mobilisées dans le discours du sujet communiquant. Ces attitudes, ces appréciations ou évaluations portées par les formes modales de l'énoncé du locuteur sont regroupées en quatre zones sémantiques modales, comme elles apparaissent dans le schéma de la modalisation discursive : zone modale ontologique, zone modale de jugement de vérité, zone modale axiologique et zone modale des valeurs finalisantes.

\section{La zone modale ontologique}

Les valeurs ontologiques renvoient aux valeurs existentielles qui rendent compte de l'existence du monde naturel et social représentés dans et par le discours. Dans cette zone modale s'inscrivent les valeurs aléthiques et les valeurs déontiques.

Les valeurs modales aléthiques correspondent à l'attitude modale de l'appréhension « du fonctionnement des lois naturelles »(Galatanu, p. 94). Au même titre que pour les modalités prépositionnelles de Parret (cité par Darrault, 1976, 6), notamment les aléthiques, la base de ces valeurs est le terme nécessaire, les autres termes se définissent en niant ce terme de départ, comme suit :

Nécessaire --------------------- Contingent (non-nécessaire) Possible Impossible (non nécessaire que non) (nécessaire que non)

De ce fait, ces valeurs modales correspondent à : $<$ nécessaire $>$, <impossible $>$, <possible $>$, <aléatoire $>$ que l'on peut représenter, à partir de Galatanu (2003), comme suit : 
Valeurs aléthiques : <nécessaire>

\begin{tabular}{|c|c|}
\hline $\begin{array}{c}\text { <nécessaire> } \\
\text { devoir être }\end{array}$ & $\begin{array}{c}\text { <impossible> } \\
\text { ne pas devoir être } \\
\text { <nécessaire > } \\
\text { <devoir ne pas être> }\end{array}$ \\
\hline $\begin{array}{c}\text { < nécessaire > } \\
\text { ce qui n'est pas nécessaire } \\
\text { <possible> }\end{array}$ & $\begin{array}{c}<\text { nécessaire }> \\
\text { <ne pas devoir être }>\end{array}$ \\
\hline
\end{tabular}

Les valeurs déontiques portent sur les normes ou règles sociales. Ces valeurs renvoient à l'organisation sociale, aux conventions que la société a créées et imposées pour assurer son bon fonctionnement. Ces valeurs se structurent sur la base du terme obligatoire, tel qu'il est présenté ci-dessous :

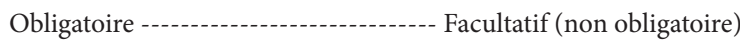
Permis (non obligatoire que non) --------- Interdit (obligatoire que non)

D'où l'organisation de ces valeurs comme suit : $<$ obligatoire $>,<$ interdit $>,<$ permis $>,<$ facultatif $>$ (Galatanu, 2003). La figure suivante rend compte de ces valeurs :

Valeurs déontiques : <obligatoire> : normes ou règles sociales

\begin{tabular}{|c|c|}
\hline $\begin{array}{c}\text { <obligatoire> } \\
\text { <devoir faire> }\end{array}$ & $\begin{array}{c}\text { <obligatoire }> \\
<\text { interdit }>\end{array}$ \\
\hline $\begin{array}{c}\text { < obligatoire > } \\
\text { <permis }>\end{array}$ & $\begin{array}{c}\text { < obligatoire }> \\
\text { <facultatif }>\end{array}$ \\
\hline
\end{tabular}

\section{La zone modale des valeurs de jugement de vérité}

Les valeurs modales qui composent cette zone concernent aussi bien les valeurs épistémiques que doxologiques. Leur convocation dans le discours permet au sujet pensant de construire une représentation du monde. Les premières concernent le certain, l'incertain, le probable, l'improbable, le médiatif et le non médiatif. Les valeurs épistémiques portent sur le savoir, la connaissance et la certitude qu'en a le sujet et de ce fait, elles sont moins subjectives que les doxologiques.
Les valeurs modales épistémiques ont pour base la valeur " certain » et au moyen de la négation on obtient les autres valeurs du carré, à savoir :

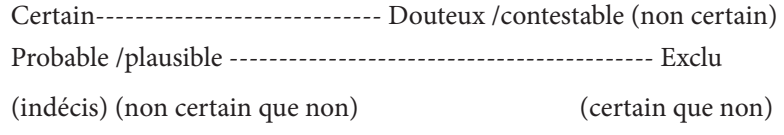

Ainsi, à partir de ce qui vient dêtre exposé, on obtient le carré des valeurs modales épistémiques suivant :

Valeurs épistémiques

\begin{tabular}{|c|c|}
\hline <certain $>$ & $<$ certain $>$ \\
$<$ certain $>$ & $<$ exclu $>$ \\
\hline$<\sim$ certain $>$ & $<\sim$ certain $>$ \\
$<$ probable $>$ & $<$ incertain $>$ \\
\hline
\end{tabular}

Les valeurs doxologiques, moins objectives que les épistémiques, se relient aux croyances et s'actualisent à travers le verbe croire / ne pas croire.

Bien que pour certains, la vérité ne se fonde pas seulement sur des certitudes, sur le savoir, mais aussi sur ce que lon croit par rapport aux autres et au monde, les valeurs doxologiques s'opposent en quelque sorte aux épistémiques. Soit les croyances sopposent aux savoirs, soit elles font partie d'un stade préalable, d'un échelon, peut-être, nécessaire dans le développement et la consolidation de ces savoirs. Ainsi, Tardif (1988, p. 152) affirme que «Opinions et idées reçues, représentations courantes et apparemment crédibles, évidences du sens commun, préjugés, solutions spontanées, réponses toutes faites, prénotions, etc., constituent ce qu'on appelle traditionnellement la doxa, terme grec signifiant à peu près opinion, et qu'on oppose à l'épistémé, c'est-à-dire au savoir scientifique. »

\section{La zone modale axiologique}

La zone modale de l'axiologique, moins étudiée par la linguistique des modalités et nétant pas l'objet de la logique modale (Galatanu, 2003b, 95), coïncide, quant aux valeurs modales quelle recouvre, avec le système de valeurs. Cette zone modale correspond à l'idée de préférence, de rupture et permet donc 
de porter des évaluations sur le vécu de l'homme, sur son expérience. Les formes modales véhiculant ces valeurs portent aussi bien sur l'individuel que sur le collectif. Dans l'axiologique, s'inscrivent les valeurs suivantes :

Ethiques-morales : ce champ de valeurs se structure autour des pôles $<$ bien $>$ et $<$ mal $>$

Esthétiques : ces valeurs renvoient à ce qui résulte agréable au sujet parlant et sont en rapport avec les conceptions de beauté et de laideur. Cet ensemble de valeurs correspond à la logique binaire $<$ beau $>$ et $<$ laid $>$.

Pragmatiques : renvoient à l'action et aux résultats obtenus en comparaison des buts fixés et des moyens investis. Ces valeurs correspondent aux logiques binaires $\langle$ utile $><$ inutile $\rangle$, $<$ rentable $>$ $<$ non rentable $>$, $<$ efficace $><$ inefficace $>$.

Intellectuelles : concernent l'intérêt que peut porter le sujet parlant envers la réalité et s'appuient sur l'activité cognitive qui permet de juger, d'estimer quelque chose comme $<$ intéressant $><$ inintéressant $>$.

Hédoniques-affectives : ces valeurs renvoient à l'affectivité du sujet communiquant et concerne le plaisir, le déplaisir, les sentiments, les émotions. Ces valeurs sont représentées par $<$ heureux $>$ $<$ malheureux $>$.

\section{La zone modale des valeurs finalisantes}

Les valeurs finalisantes, ou de jugement d'intentionnalité, se situent à l'extrême le plus subjectif de l'axe de lévaluation que peut porter le sujet. Ces valeurs désignent l'intention, le désir, la volonté et le souhait. Cette zone recouvre les valeurs volitives (le vouloir) et les désidératives (le désir, le souhait).

Les valeurs volitives sont en rapport avec la capacité individuelle à se fixer des buts à atteindre, de parvenir à une amélioration de soi ou, du moins, à une transformation. Ces valeurs se fondent sur la volonté et la prise de décisions.

Les zones sémantiques modales exposées cidessus se situent sur un axe d'appréciation ou d'évaluation dont l'un des pôles soriente vers les valeurs les plus objectives et l'autre vers les valeurs les plus subjectives. Le schéma suivant illustre ce mouvement des valeurs modales portées par les formes modales que le sujet parlant mobilise dans son discours :

\section{La fonction discursive}

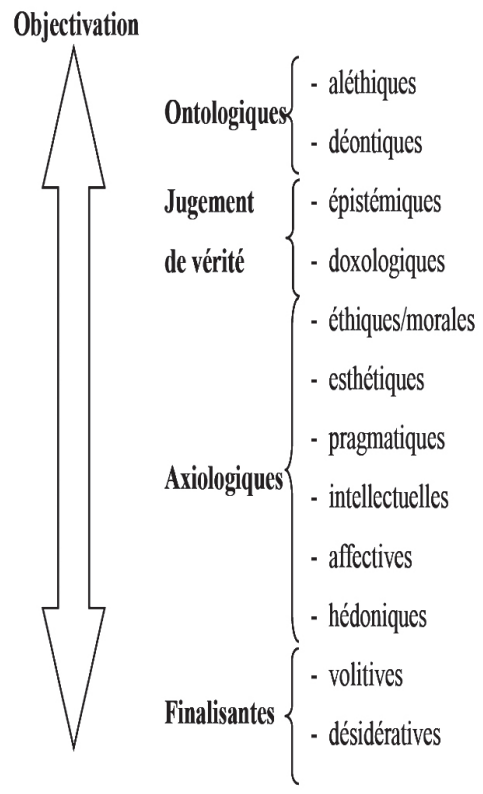

Subjectivation

La fonction discursive (Galatanu, 1996d)

Dans ce cadre constitué par les modalités, Galatanu (2003b, p. 99), à partir des développements théoriques de l'argumentation dans la langue (Anscombre et Ducrot, 1983), des topoï et des formes topiques (Anscombre (éd), 1995) et de la sémantique argumentative (Ducrot et Carel, 1999), signale que le non-modal n'existe pas dans le discours car tout énoncé est argumentatif et donc, il existe toujours une prise de position par le sujet parlant dans des conditions discursives " normales ». Ceci met en relief l'importance des valeurs modales portées par la signification des entités linguistiques. Lorsque ces unités linguistiques sont mobilisées, les mécanismes langagiers qui supportent la fonction argumentative du sens discursif sont différents d'une entité lexicale à l'autre, id est "'l'autorisation' de certains enchaînements discursifs et 'l'interdiction' d'autres 
enchaînements n'est pas inscrite dans la signification lexicale de la même façon, ni au même niveau, pour toutes les unités lexicales (Galatanu, 2000, 2002). » (Galatanu, p. 99).

En établissant la différence entre les mécanismes sémantico-discursifs et les mécanismes pragmaticodiscursifs dans la construction du sens, celui-ci marqué par son caractère argumentatif et modal, Galatanu justifie l'étude de la modalisation. C'est en raison de cette approche qu'elle défend qu'elle s'inscrit dans la filiation de la sémantique argumentative. (Galatanu, 2003b). Par conséquent, Galatanu propose le modèle de description de la signification lexicale qui permet de prouver que «le 'potentiel modal' n'est pas inscrit de la même façon dans toutes les entités linguistiques»(2003b). Voilà donc la justification de l'analyse de certaines de ces unités lexicales comme étant des formes modales, autrement dit " des entités linguistiques porteuses de valeurs modales ». Ce modèle, dit-elle, permet aussi de montrer que le discours peut transformer le potentiel argumentatif des entités mobilisées, c'està-dire que le « discours ne préserve pas toujours les valeurs inscrites dans les significations des entités linguistiques et qu'il peut reconstruire, par des mécanismes sémantico-discursifs ou pragmaticodiscursifs le potentiel argumentatif des mots qu'il mobilise » (Galatanu, pp. 99-100). Au passage, il faut ajouter que ce modèle est aussi en mesure d'analyser les phénomènes discursifs inférentiels qui ne font pas partie de l'objet de la sémantique argumentative.

Etant constitué d'une opération mentale d'association de représentations du monde « dans un bloc signifiant » et d'une opération mentale de sélection d'une relation entre ces représentations, lacte discursif englobe deux phénomènes discursifs de production de sens :

le phénomène sémantico-discursif, qui repose sur « un degré de stabilité très élevé de cette association des représentations, association conventionnalisée linguistiquement, id est présente dans la signification des entités linguistiques ", est l'objet le plus important pour la sémantique argumentative. (Galatanu, p. 100) le phénomène pragmatico-discursif désigne des « associations de représentations proposées par l'acte discursif, dans la singularité de son contexte. » (Galatanu, p. 100)

Or, si les déploiements des associations et des sélections du lien entre les représentations ne sont pas présents dans les séquences discursives, deux types de calculs sont donc indispensables :

le calcul du sens à partir de l'orientation argumentative inscrite dans la signification des entités lexicales

le calcul inférentiel, s'appuyant sur la mobilisation d'informations contextuelles ou stockées dans la mémoire (Galatanu, p. 101)

Ce qui précède permet de comprendre, d'un côté, que dans certaines séquences discursives à un énoncé à visée argumentative axiologiquement positive ou négative lui suffit, pour sa compréhension et/ou explication, la présence des entités lexicales qui le composent dans la mesure où ces dernières ont inscrit dans leur signification une valeur axiologique (positive ou négative). Et, d'un autre côté, cela permet de comprendre que dans d'autres énoncés, la visée argumentative, axiologiquement positive ou négative, dépend du contexte dans lequel ces énoncés ont lieu en raison de l'absence d'une prise de position dans la signification de l'entité lexicale.

De même, il existe des entités linguistiques axiologiquement monovalentes, comme crime, viol, par exemple, et d'autres entités axiologiquement bivalentes, telles que guerre, grève... les secondes pouvant être orientées vers des conclusions axiologiquement positives ou négatives suivant le co-texte ou le contexte. C'est à partir de la distinction entre ces deux types d'entités qu'il est obligatoire de proposer une théorie sémantique qui puisse « rendre compte du niveau d'inscription de la valeur axiologique dans la signification des mots » (Galatanu, p. 101). La théorie pouvant faire apparaître ces caractéristiques inscrites dans la signification des unités lexicales et proposer un modèle de description de la signification lexicale est la Sémantique des Possibles Argumentatifs. 
Il nous reste à préciser que cette approche a accordé un statut linguistique et un statut discursif au concept de modal.

\section{Les fonctions discursives : objectivation et subjectivation}

En fonction des valeurs modales que convoquent les formes modales insérées dans leurs discours, les sujets communiquants rendent compte d'un degré plus ou moins marqué d’objectivation et plus ou moins marqué de subjectivation. En ce sens, Galatanu (1997a), signale deux types de mouvements dans le discours: un mouvement d'objectivation et un mouvement de subjectivation. Ces deux mouvements s'activent sur l'axe des zones modales, ils sorientent vers la zone ontologique ou vers la zone de valeurs finalisantes et sont en rapport avec les formes modales qui véhiculent les valeurs ou, mieux, qui apparaissent dès que le sujet transforme la langue en discours, notamment dans ces formes modales. Le rôle du spécialiste de l'analyse linguistique du discours consistera à mettre en place un carrefour qui rende compte de la manière dont le locuteur objective ou subjective son discours au moyen de formes modales porteuses de valeurs modales. En schématisant ce double regard du chercheur, il devrait mener à bien une analyse suivant ce schéma :

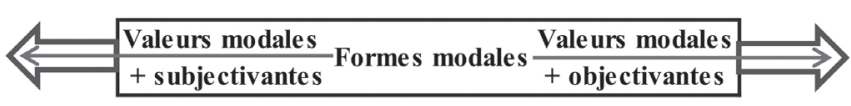

Les deux trajectoires, faisant partie d'un axe, s'activent suivant l'intérêt du sujet parlant de s'occulter ou d'apparaître clairement dans son discours. A ce sujet, Kerbrat-Orecchioni (1980, p. 70) affirme que les unités lexicales sont subjectives car elles ne fournissent aucun analogon de la réalité, elles ne sont pas que des "symboles substitutifs et interprétatifs des « choses » . Ces entités lexicales découpent et organisent le monde «à leur manière » et, en reprenant Lee Whorf, les sujets communiquants découpent l'univers référentiel à partir des possibilités existantes dans les langues.
A cela s'ajoute le rôle de la communauté qui agirait comme un inconscient linguistique collectif déterminant les descriptions que ses membres font du monde. Kerbrat-Orecchioni s'intéresse surtout à l'usage individuel que les sujets font de la langue et non à l'action linguistique collective. Dans cet ordre d'idées, elle affirme que devant lénonciation, le sujet parlant choisit de produire un discours plus objectivant ou plus subjectivant: " [...] il a en gros le choix entre deux types de formulations : le discours « objectif », qui sefforce de gommer toute trace de l'existence d'un énonciateur individuel ; le discours «subjectif » dans lequel l'énonciateur s'avoue explicitement $[\ldots]$ ou se pose implicitement [...] comme la source évaluative de l'assertion ». Le fait que les entités lexicales soient plus ou moins porteuses de subjectivité dans la langue n'empêchent pas le sujet parlant de puiser dans le réservoir linguistique et, en fonction de la culture, du contexte, entre autres, il décide de s'en servir en vue de présenter un discours plus « transparent» ou plus « opaque », id est plutôt visible ou plutôt effacé sur le plan de l'objectivation ou de la subjectivation. (Kerbrat-Orecchioni, p. 71)

L'implication du sujet parlant (objectivation ou subjectivation) peut être exprimée de manière explicite au moyen du mécanisme discursif de transparence. Mais le sujet communiquant peut aussi, au moyen du mécanisme d'opacité, se replier afin de voiler le degré d'implication objectivante ou subjectivante. Introduisant ces mécanismes utiles à l'analyse linguistique du discours, Galatanu (2000a) montre le rapport des formes modales et de leurs valeurs modales qui se croisent pour rendre compte de l'implication du sujet parlant. A partir des aspects précédents Galatanu (2000a) définit les mécanismes qui en résultent comme suit :

- un mécanisme reposant sur une correspondance entre la subjectivation des contenus par les valeurs convoquées et la subjectivation par les formes linguistiques mobilisées.

- un mécanisme s'appuyant sur la concordance de lobjectivation des contenus par les valeurs convoquées et l'objectivation par les formes linguistiques mobilisées. 
- un mécanisme discursif d’objectivation explicite.

- un mécanisme reposant sur la discordance entre la subjectivation des contenus par les valeurs convoquées et l'objectivation par les formes mobilisées: un mécanisme discursif d'occultation de la subjectivation; le mécanisme s'appuyant sur la discordance entre l'objectivation des contenus par les valeurs convoquées et la subjectivation par les formes mobilisées : mécanisme d'occultation de l'objectivation.

Galatanu (1996d) propose un schéma concrétisant les mécanismes ci-dessus qui résultent de croiser les formes modales et les valeurs qu'elles portent. Voici donc la schématisation :

\section{Les mécanismes discursifs}

\begin{tabular}{|c|c|c|}
\hline & \multicolumn{2}{|c|}{ Formes linguistiques } \\
\hline \multirow[t]{5}{*}{ Valeurs } & MD : mécanismes discur & s: A, B, C, D \\
\hline & A & B \\
\hline & $\begin{array}{l}\text { Valeurs objectivantes et formes } \\
\text { objectivantes: objectivité } \\
\text { transparente }\end{array}$ & $\begin{array}{l}\text { Valeurs objectivantes et } \\
\text { formes subjectivantes: } \\
\text { révélation }\end{array}$ \\
\hline & C & D \\
\hline & $\begin{array}{l}\text { Valeurs très subjectivantes dans } \\
\text { les formes très objectivantes: } \\
\text { MD d'occultation du mouvement } \\
\text { de subjectivation : recul, repli }\end{array}$ & $\begin{array}{l}\text { Valeurs subjectivantes et } \\
\text { formes subjectivantes: } \\
\text { subjectivité transparente }\end{array}$ \\
\hline
\end{tabular}

On peut observer que les pôles attribuables à l'ensemble de ces quatre mécanismes sont formés, d'une part, par les MD A en raison de la coïncidence des formes et des valeurs objectivantes, ce qui produit l'objectivité transparente. D’autre part, les MD $\mathrm{D}$ rendent compte de la coïncidence de formes et valeurs subjectivantes en entraînant l'existence d'une subjectivité transparente. Dans les points intermédiaires de l'axe réunissant ces mécanismes se situent les MD B y MD C dans lesquels les formes et les valeurs mobilisées sont soit objectivantes, soit subjectivantes.
Dans le domaine de la sémantique, le programme de recherche entamé et développé par la SPA a envisagé l'identification du niveau d'inscription des valeurs modales (les " prises de position ») dans la signification lexicale. Les entités linguistiques se caractérisent parfois par la présence d'une valeur axiologique négative ou positive dans leurs stéréotypes, dans le mot vol par exemple, et on parle alors de monovalence. Parfois, elles se caractérisent par une bivalence ou polarité, id est l'inscription de valeurs axiologiques positives et négatives, comme dans le cas du mot guerre, dans leurs possibles argumentatifs (Galatanu 2003, p. 103). Lorsqu'on est face à des entités lexicales bivalentes et qui sont mobilisées dans le discours, c'est la contamination discursive, avec les stéréotypes des mots du contexte, qui sélectionne et active l'un o l'autre des pôles axiologiques, et stabilise une orientation argumentative.

De ce point de vue, pour la SPA sont modales les entités lexicales qui comptent «[...] parmi les éléments de leurs stéréotypes ou même de leurs noyaux, une ou plusieurs valeurs modales. » (Galatanu, p. 104).

Dans le domaine de l'analyse linguistique du discours, le programme de recherche vise «l'activation des valeurs modales axiologiques pour les nominaux bivalents et sur le renforcement ou l'affaiblissement, voire l'interversion des valeurs portées par les nominaux axiologiquement monovalents, et, ipso facto, sur la re-construction discursive des systèmes de valeurs »(Galatanu, p. 103). Dans ce sens, d'un point de vue du sens pragmatique ou discursif, la modalisation correspond à la " mobilisation d'entités linguistiques modales » dans un processus de contamination qui, de par l'interaction des stéréotypes, contribue à la confirmation, la modification et même l'inversion des valeurs inscrites dans les mots.

\section{Les formes modales}

Ces formes modales correspondent aux entités lexicales de la langue. Elles portent des valeurs modales dans leur signification. A ce sujet, Galatanu établit que « sur le plan sémantique, nous considérons comme modales les entités linguistiques qui ont 
parmi les éléments de leurs stéréotypes ou même de leurs noyaux, une ou plusieurs valeurs modales. » (Galatanu, p. 104). La mobilisation de ces formes, qui à leur tour convoquent des valeurs modales, est à la base des fonctions discursives que Galatanu présente comme des " processus dévaluation du monde construit par le discours. (Galatanu, p. 93). Une autre caractéristique de ces formes modales consiste à ce que le "le potentiel modal " est inscrit différemment dans toutes les entités lexicales. De même, les valeurs inscrites dans ces entités linguistiques peuvent être modifiées en raison de l'action de mécanismes sémantico-discursifs et pragmaticodiscursifs, ce qui va reconstruire leur potentiel argumentatif. D'autre part, les formes modales peuvent être monovalentes o bivalentes. Les premières inscrivent dans leur noyau ou dans leurs stéréotypes, l'un des pôles axiologiques (positif ou négatif). Les secondes portent dans leurs strates les deux pôles et l'un d'eux s'active dans la mobilisation discursive. Galatanu fait remarquer que " La distinction entre des entités linguistiques axiologiquement monovalentes ou axiologiquement bivalentes exige [...] une théorie sémantique susceptible de rendre compte du niveau d'inscription de la valeur axiologique dans la signification des mots. » (Galatanu, p. 101)

A présent, en revenant aux mécanismes d’objectivation et de subjectivation, ainsi quaux valeurs modales, tous les deux abordés précédemment, il est important de présenter deux modalités autour desquelles s'organisent les formes modales. Il s'agit des modalités de RE et des modalités de DICTO.

Modalités de RE : au Moyen-âge, comme indiqué ailleurs, Thomas d'Aquin a opposé cette modalité à la modalité de DICTO. A titre d'information, RE est traduit comme "à propos de la chose", tandis que DICTO signifie "à propos de ce qui est dit ». La modalité de RE regroupe les étiquettes nominales, les étiquettes verbales et l'ensemble de leurs modificateurs. Ces derniers concernent les caractérisants qui se lient aux étiquettes nominales, des modificateurs de verbe se reliant aux étiquettes verbales. Quant à l'axe objectivation/subjectivation, les étiquettes nominales apparaissent comme plus objectivantes, alors que les modificateurs sorientent vers la subjectivation.

Modalités de DICTO : plus marquées subjectivement que les modalités précédentes, elles concernent les verbes à usage modal, les adjectifs opérateurs de phrase et l'adverbe modalisateur de phrase.

Dans le schéma ci-dessous, Galatanu croise les zones sémantiques modales avec les formes modales (modalités de RE et de DICTO), ainsi que les axes objectivation/subjectivation, ce qui favorise une meilleure appréciation de l'ampleur des relations dans le cadre de l'analyse du discours et de la sémantique argumentative :

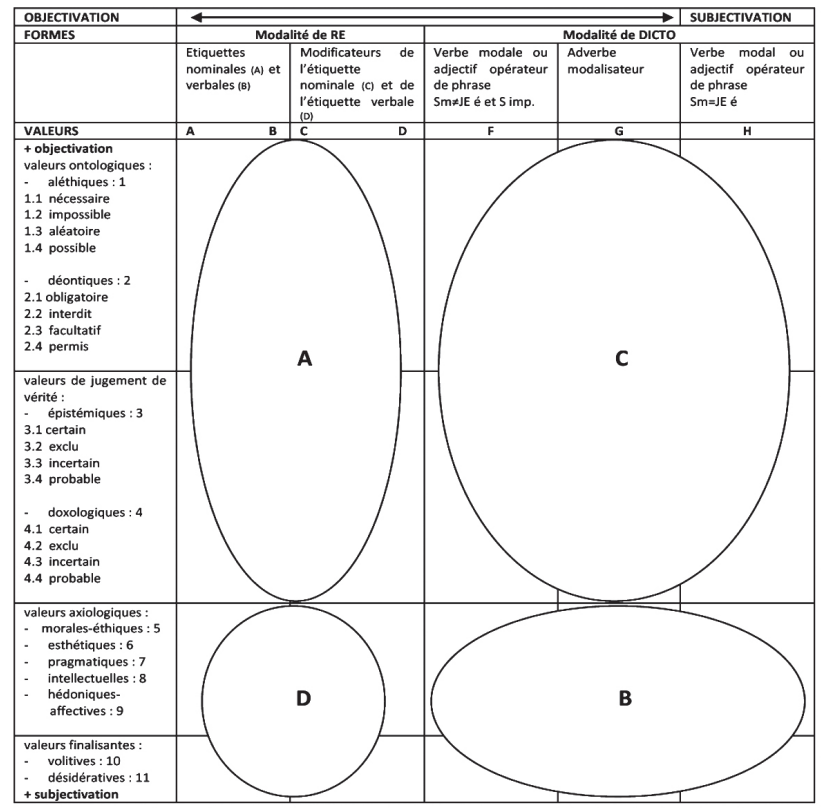

Le croisement des valeurs et des formes modales (Galatanu, 2002a)

Dans le tableau ci-dessus, le croisement de l'axe objectivation/subjectivation vertical avec l'axe d'objectivation/subjectivation horizontal, id est les zones sémantiques modales et les formes modales respectivement, permet d'obtenir les mouvements et les mécanismes discursifs suivants :

- A : mouvement dobjectivation en concordance avec un mouvement d'objectivation par les formes linguistiques. Il s'agit d'un mécanisme discursif d'explicitation de l'objectivation.

- $C$ : mouvement d'objectivation en discordance avec un mouvement de subjectivation par les 
formes linguistiques. Il s'agit d'un mécanisme discursif d’opacité de l'objectivation.

- D : mouvement de subjectivation en discordance avec un mouvement d'objectivation par les formes linguistiques. Il s'agit d'un mécanisme discursif d'opacité de subjectivation.

- B: mouvement de subjectivation en concordance avec un mouvement de subjectivation par les formes linguistiques. Il s'agit d'un mécanisme discursif d'explicitation de la subjectivation. (Galatanu, 2002a).

Selon Galatanu, en tant que termes porteurs de valeurs modales, les entités linguistiques sont des formes modales agissant comme des véhicules de l'orientation argumentative. Dans cet ordre d'idées, elle conditionne la discussion sur les valeurs à la discussion sur « le problème de leur énonciation et de leur acceptation, qui passe par une procédure communicationnelle de conviction " (2002b). Elle ajoute que la procédure communicationnelle est assurée sur le plan discursif par la mise en œuvre, par des mécanismes argumentatifs, des valeurs contenues durablement dans la signification de certaines entités lexicales ou des mots renvoyant au « « fait social » $[. .$.$] ou des mots désignant les$ pôles de la zone sémantique de l'axiologie [...] que je définirai comme des universaux sémantiques. » (Galatanu, 2002b)

En guise de conclusion, les aspects abordés brièvement dans ce texte mettent en évidence l'importance et la pertinence de la SPA pour les sciences du langage, pour l'analyse linguistique du discours et pour la signification lexicale. Ses apports à la didactique de l'enseignement des langues étrangères sont aussi significatifs, d'autant plus que cette orientation insiste sur une conception différente qui sécarte de l'idée référentialiste de l'enseignement des langues. Par conséquent, les considérations abordées invitent à faire des recherches dans le domaine en vue de faire avancer le modèle et de mieux saisir le discours, la signification, le sens et les implications pour la didactique des langues étrangères.

\section{Bibliographie}

Anscombre, J.-C. et Ducrot O. (1983). Largumentation dans la langue. Liège : Pierre Mardaga.

Anscombre J.-C. (1995). La théorie des topoï: Sémantique ou rhétorique? In Hermès, vol. 15, 185-198.

Benveniste, E. (1970). Lappareil formel de l'énonciation. In Langages, vol 5, 17, 12-18.

Benveniste, E. (1966). De la subjectivité dans le langage [En ligne], 258-266.

Disponible sur http://latina.phil2.uni-freiburg.de/ raible/Lehre/2006/Materialien/Benveniste.pdf

Carel, M. et Ducrot, O. (1999). Le problème du paradoxe dans une sémantique argumentative. In Langue Française, n ${ }^{\circ}$ 23, 6-26.

Charaudeau, P. (1992). Grammaire du sens et de l'expression. Paris : Hachette Education.

Chu, X. (2008). Les verbes modaux du français. Paris : Ophrys.

Darrault, Y. (1976). Présentation. In Langages, vol. $10 n^{\circ} 43,3-9$.

Dervin, F. (2009). Approches dialogiques et énonciatives de l'interculturel : pour une didactique des langues et de l'identité mouvante des sujets. In Synergies Roumanie, 4, 165-178

Ducrot, O. (1988). Polifonía y argumentación. Cali: Universidad del Valle.

Galatanu, O. (1996d). Analyse des discours et approche des identités. In Revue Education permanente, 128, 45-61.

Galatanu, O. (1997). Les Argumentations du discours lyrique. Ecriture poétique moderne: le narratif, le poétique, l'argumentatif. Nantes : CRINI, Université de Nantes.

Galatanu, O. (1999a). Le phénomène sémanticodiscursif de déconstruction-reconstruction des topoï dans une sémantique argumentative intégrée. In Langue Française, 123, 41-51.

Galatanu, O. (1999b). Argumentation et analyse du discours. In Jalons 2, 41-54.

Galatanu, O. (2000). La Reconstruction du système de valeurs convoquées et évoquées dans le discours médiatique. In Actes du XXIIe congrès international de linguistique et philologie romanes, VII, 251-258. 
Galatanu, O. (2000a). Signification, sens et construction discursive de soi et du monde. In Barbier, J.-M. et Galatanu, O. (éd.), Signification, Sens, formation. Paris : PUF.

Galatanu, O. (2002). Le concept de modalité : les valeurs dans la langue et dans le discours. In Les valeurs, Séminaire Le lien social, 17-32.

Galatanu, O. (2002a). La Dimension axiologique de l'argumentation. Les facettes du dire. In Carel, M. (éd.), Hommage à Oswald Ducrot. Paris : Kimé.

Galatanu, O. (2002b). Les valeurs, Maison des Sciences de l'homme Ange Guépin. In Le Roy, F. (éd.) et Galatanu O. (coord.). Séminaire Le Lien Social.

Galatanu, O. (2003a). La Sémantique des possibles argumentatifs et ses enjeux pour l'analyse du discours. En El texto como encrucijada: estudios franceses y francófonos, 2, 213-226.

Galatanu, O. (2003b). La construction discursive des valeurs. In Barbier J.-M. (dir.), Valeurs et activités professionnelles. Séminaire du Centre de Recherche sur la Formation du CNAM. Paris : L'Harmattan.

Galatanu, O. (2005a). Analyse du discours. In Diversité Ville, école, intégration [En ligne]. Disponible sur http://www.cndp.fr/archivage/ valid/70174/70174-10778-13726.pdf

Galatanu, O. (2005b). La "stéréophagie » un phénomène discursif de déconstruction-

reconstruction de la signification lexicale. In Les Actes du Colloque International «Représentation du sens linguistique III-RSL III, 189-207.

Galatanu, O. (2006). Le cinétisme de la signification lexicale. In Barbier, J.M. et Durand, M. (éd), Sujets, activité, environnement. Paris : Presses Universitaires de France.

Galatanu, O. (2007). L'expression de l'affect dans l'interaction en situation de contact des langues et cultures: à l'interface des compétences sémantique et pragmatique [en ligne]. In Acte du colloque Les enjeux de la communication interculturelle, Compétence linguistique, compétence pragmatique, valeurs culturelles. Disponible sur : http://www.msh-m.fr/diffusions/editionsen-ligne/Actes-en-ligne/Les-enjeux-de-lacommunication/L-expression-de-l-affect-dans-1
Galatanu, O. (2007a). Sémantique des possibles argumentatifs et axiologisation discursive. In Bouchard, D. et Evrard, J. (éd.), Représentation du sens linguistique 2. Louvain-La-Neuve : Duculot De Boeck.

Galatanu, O. (2007b). Pour une approche sémanticodiscursive du stéréotypage à l'interface de la sémantique théorique et de l'analyse du discours. Stéréotypage, stéréotypes : fonctionnements ordinaires et mises en scène. In Boyer, H. (éd.), Langue(s), Discours, tome 4. Paris : L'Harmattan.

Galatanu, O. (2008). La construction discursive des représentations de la langue française et de la francophonie chez les futurs enseignants du FLE en Asie-Pacifiques. In Actes du Premier Congrès Régional Asie-Pacifique, Le français, la francophonie et la francophilie en Asie-Pacifique : spécificités et interrogations.

Galatanu, O. (2008). L'interface linguistique-culturel et la construction du sens dans la communication didactique. In Signes, Discours et Sociétés [en ligne]. Interculturalité et intercommunication. Disponible sur http://revue-signes.info/ document.php?id=263 ISSN 1308-8378

Galatanu, O. (2009a). L'Analyse du Discours dans la perspective de la Sémantique des Possibles argumentatifs: les mécanismes sémantico-discursifs de construction du sens et de reconstruction de la signification lexicale. In Cahiers du Laboratoire de Recherche sur le Langage, 49-68.

Galatanu, O. (2009b). Les incidences sémantiques des déploiements argumentatifs dépendants du co-(n)texte de production du discours. In Mémoires de la Société Néophilologique de Helsinki LXXVIII, 391-404.

Galatanu, O. et Nikolenko, V. (2009). Acquisition du lexique de la zone sémantique de l'axiologique: Le cas des apprenants avancés. In Galatanu, O., Pierrard, M. et Van Raemdonck, D. (dir.), Construction du sens et acquisition de la signification linguistique dans l'interaction. Bruxelles: P.I.E. Peter Lang.

García Negroni, M. M. y Tordesillas Colado, M. (2001). Enunciación en la Lengua: de la deixis a la polifonía. Madrid: Gredos.

Ignatieva Chaillou, M. (2011). Le phénomène d’axiologisation discursive de la signification lexicale Texte imprimé le cas de harmonisation dans le 
cadre de l'Union Européenne. (Recuperado 05 agosto de 2012 http://archive.bu.univ-nantes. fr/pollux/show.action?id=8019845b-cf25-42369278-3471f9a3838d).

Kerbrat-Orecchioni, C. (1980). Lénonciation de la subjectivité dans le langage. Paris : Armand Colin.

Maingueneau, D. (1976). Initiation aux méthodes de l'analyse du discours. Paris : Hachette, 191p.

Maingueneau, D. (1984). Genèses du discours. Bruxelles : Mardaga.

Maingueneau, D., (2005). Lanalyse du discours et ses frontières. In Marges linguistiques, 9. Disponible sur http://dominique.maingueneau.pagespersoorange.fr/conclusion2.html

Marie, V. (2008). Conception de la Francophonie/ Francophilie en Moldavie : représentations discursives et stéréotypes. (Recuperado 05 agosto de 2012, http://archive.bu.univ-nantes.fr/pollux/ show.action?id=6e6af8e7-b6ba-4e3a-854ca61945ccc14f).

Nikolenko, V. (2011). Le cinétisme de la signification lexicale dans la zone sémantique de l'axiologique : le cas du lexique dans l'enseignement du FLE en France. (Recuperado 05 agosto de 2012, http://archive.bu.univ-nantes.fr/pollux/ show.action?id=2544ed3d-5699-47d4-a70169273f74b923).

Nyckees, V. (2007). La cognition humaine saisie par le langage : De la sémantique cognitive au médiationnisme [en ligne]. Corela, Numéros spéciaux, Cognition, discours, contextes. Disponible sur http://edel.univ-poitiers.fr/corela/ document.php?id=1659

Perret, M. (1994). Lénonciation en grammaire du texte. Paris : Nathan.

Pescheux, M. (2003). Construction de sens et modèle argumentatif de la signification lexicale : une formulation de stéréotypes "lexicaux». En El texto como encrucijada: estudios franceses $y$ francófonos [en ligne], 2, 259-270. Disponible sur http://dialnet.unirioja.es/servlet/fichero_articulo? codigo $=1011559$ \&orden $=74673$

Putnam, H. (1975). The meaning of " meaning ». In Philosophophical papers, 2, Cambridge University Press.
Rastier, F. (2001). Sémantique et recherches cognitives. Paris : PUF.

Rastier, F. (2005). Sémiotique du cognitivisme et sémantique cognitive: Questions d'histoire et d’épistémologie. In Texto! [en ligne]. Disponible sur http://www.revue-texto.net/Inedits/Rastier/ Rastier_Semantique-cognitive.html

Rastier, F. (2006). De la signification lexicale au sens textuel : éléments pour une approche unifiée. In Texto! [en ligne], XI, 1. Disponible sur http://www.revue-texto.net/Inedits/Rastier/ Rastier_Signification-lexicale.html

Ruiz Vega, J. (2011). La mobilisation discursive des valeurs sociales chez les futurs enseignants de FLE en Colombie. (Recuperado 05 agosto de 2012, http://archive.bu.univ-nantes.fr/pollux/ show.action?id=0d304f4b-cd64-4712-ade2$2 \mathrm{~d} 0 \mathrm{f0058d} 3 \mathrm{e} 8$ ).

Tamba-Mecz, I. (1988). La semántica. México D.F.: Fondo de Cultura Económica.

Tardif, M. (1988). Le chercheur et les contraintes doxologiques. In Revue des sciences de léducation, vol. 14, 2, 149-163.

Le Nouveau Petit Robert de la Langue française (2009). Paris : Le Robert.

Lexis Larousse de la Langue Française (2005). Varese : Larousse. 